\title{
Numerical Evaluation of the Vibration Reduction Index for Structural Joints
}

\author{
Jaime RAMIS(1), Enrique SEGOVIA(1), Jesús ALBA ${ }^{(2)}$, Jesús CARBAJO(1) ${ }^{(1)}$ Luís GODINHO ${ }^{(3)}$ \\ (1) Department of Physics, System Engineering and Signal Theory, University of Alicante \\ Mail Box 99; 03080 Alicante, Spain; e-mail: jramis@ua.es \\ (2) Research Institute for Integrated Coastal Zone Management-IGIC, Polytechnic University of Valencia \\ Grao de Gandia 46730 (Valencia), Spain \\ (3) Department of Civil Engineering, Pólo 2 - FCTUC, University of Coimbra CICC \\ Rua Luís Reis Santos, 3030-788 Coimbra, Portugal \\ (received February 2, 2012; accepted March 29, 2012)
}

\begin{abstract}
The present paper addresses the analysis of structural vibration transmission in the presence of structural joints. The problem is tackled from a numerical point of view, analyzing some scenarios by using finite element models. The numerical results obtained making use of this process are then compared with those evaluated using the EN 12354 standard vibration reduction index concept. It is shown that, even for the simplest cases, the behavior of a structural joint is complex and evidences the frequency dependence. Comparison with results obtained by empirical formulas reveals that those of the standards cannot accurately reproduce the expected behavior, and thus indicate that alternative complementary calculation procedures are required. A simple methodology to estimate the difference between numerical and standard predictions is here proposed allowing the calculation of an adaptation term that makes both approaches converge. This term was found to be solution-dependent, and thus should be evaluated for each structure.
\end{abstract}

Keywords: Finite Element Model, vibration reduction, structural joints.

\section{Introduction}

Flanking transmission is a well-known issue that occurs on in-situ measurements of sound insulation, leading to a lower performance of separating elements between adjacent spaces. Many solutions have been developed to minimize this interference, such as the use of resilient or elastic interlayers under floating floors. The application of such solutions usually leads to systems with a more complex structure, whose behavior cannot be so easily predicted.

Several methods for predicting the influence of flanking transmissions can be found in the literature. In the late 1970's and 1980's, two reference works published by GERRETSEN $(1979,1986)$ approached the problem of flanking transmission from a practical point of view, introducing engineering procedures for the calculation of those transmissions when analyzing both the impact and airborne sound insulation. These two works have indeed lead to the development of calculation standards (EN ISO, 2000) in use for current engineering practice. In those standards, a simplified Statistical Energy Analysis (SEA) approach is used to predict sound transmission between two rooms incorporating the effects of the first order flanking paths by means of the so-called Kij coefficients. However, these standards may not be applied in a number of situations, and thus they are not completely generic. An example in airborne sound insulations evaluation (EN 12354-1) are systems incorporating lightweight partitions since these are not correctly handled as demonstrated by MAHN (2009) or BRUNSKOG et al. (2007). In fact, lightweight elements usually have higher critical frequencies when compared to heavyweight solutions, and also exhibit an orthotropic behavior, both of which are not correctly accounted for in the models proposed by the standard.

The results provided by EN 12354-1 have been analyzed in multiple works, and several other weaknesses have been identified. In the works of PEDERSEN (1995) and Metzen (1999), comparisons between computed predictions using the EN 12354-1 proposed method 
and experimental measurements allowed the authors to conclude that differences of at least $2 \mathrm{~dB}$ together with a frequency dependent difference always occurred. In a different work, CRAIK (2001) concluded that incorporating first order flanking paths, while ignoring higher order paths, lead to $5 \mathrm{~dB}$ to $10 \mathrm{~dB}$ differences. GALBRUN (2008) confirmed this finding experimentally, showing that an overestimation of the sound reduction of at least $5 \mathrm{~dB}$ occurs when neglecting higher order paths.

The limitations identified by several researchers, regardless of the accuracy of the predictions provided by the EN 12354, originated an increasing interest in the development of new procedures to broaden the applicability of standardized methods while rendering them more accurately. An example is the case of lightweight structures, for which research is being developed in the sense of extending the model and its applicability for this case, and the definition of accurate input data for the models, which can greatly influence their final output (GERretsen, 2008).

The standard limitations originated other strategies to be devised by researchers to handle the prediction of airborne and impact sound insulation in dwellings. Thus, several attempts have been made to better study the sound transmission and trying correctly to understand the phenomenon. Many of them are experimental approaches, based either on vibration measurements according to ISO 10848-1 (ISO, 2006), or near field holography techniques (MAYNARD et al., 1985; BRUTEL-VUILMET et al., 2006; 2007). In a different approach, scale models have been applied to study experimentally the acoustic behavior of different constructive solutions that can be found in the work of KLING (2008). However, scale models require special care in the definition of the model properties since the relevant relations between geometry, stiffness and mass should be similar to those of the full scale model. Additionally, small-sized instruments must be used since large microphones or accelerometers interfere with the dynamic behavior of the system.

An alternative is to make use of numerical methods to perform the analysis. Several types of methods were applied ranging from the boundary element method (BEM) to Finite Element Method (FEM) models and including mixed formulations. MALUSKI and GiBBs (2000) used a FEM model to predict the low frequency sound insulation between adjacent rooms and concluded that the sound insulation is strongly dependent on the modal behavior of the separated compartments. SAnTOS and TADEU (2002) studied the acoustic insulation provided by a single wall with elastic behavior separating two tunnels. The model included the complete transmission paths between both spaces. They concluded that the geometry, dynamic behaviour of the tunnels and the flanking vibrations have a very great influence on the sound insulation provided by the separating wall. In the works by CLASEN and LANGER (2007), a FEM formulation to simulate sound transmission including the damping mechanisms involved in the transmission phenomenon was developed. The computational results presented in that study have shown good agreement with measured results within a large frequency range.

The present paper aims at further contributing to this discussion, presenting possible strategies for improving impact sound insulation predictions of usual constructive solutions and accounting with flanking transmission. The analyzed cases consist of cross joints simulating structural configurations where significant vibration transmission occurs. In addition, the effect of a floating slab, built over a flexible material, is also studied. Three-dimensional Finite Element models are developed to evaluate the vibration reduction index in such scenarios; the obtained results are then compared with those provided by the EN 12354-2 standard trying to understand better the possible limitations of the equations proposed for different typical constructive situations.

The paper is structured as follows: in Sec. 2, a global view of the background theory is given presenting the relevant approaches collected in the EN 12354-2 standard; in Sec. 3, numerical models used for the computational analysis of the different scenarios are described and an initial comparison between numerical results and those calculated based on the standard is presented; in Sec. 4, a discussion of the result differences is performed and a strategy to quantify them is proposed; finally Sec. 5 describes the main conclusions of this paper.

\section{Background theory}

According to EN 12354 the flanking transmission can be determined making use of the vibrational reduction index concept. This standard provides empirical formulas recognized for some constructive solutions and a limited set of designs with rigid or elastic elements inserted. The approach proposed in the standard is based on the knowledge of the so-called $K_{i j}$ vibrational reduction index, which is related to the vibrational power transmission through a junction between structural elements, normalized in order to make it scale invariant. It can be determined experimentally through laboratory tests according to ISO 10848-1.

The experimental evaluation of the vibrational reduction index is performed normalizing the joint averaged difference of the velocity levels, $\left(\overline{D_{v, i j}}\right)$, with the junction length and the equivalent absorption length of the connected elements according to the equation:

$$
K_{i j}=\frac{D_{v, i j}+D_{v, j i}}{2}+10 \log \frac{l_{i j}}{\sqrt{a_{i} a_{j}}}[\mathrm{~dB}],
$$


$D_{v, i j}$ is the difference of the velocity levels between the $i$ and $j$ elements in $\mathrm{dB}$, when the $i$ element is excited,; $l_{i j}$ is the common coupling junction length of the $i$ and $j$ elements in meters; $a_{i}$ and $a_{j}$ the equivalent absorption lengths of the $i$ and $j$ elements, respectively, both in meters. The equivalent absorption length can be calculated using the following equation:

$$
a=\frac{2.2 \pi^{2} S}{c_{o} T_{s}} \sqrt{\frac{f_{\mathrm{ref}}}{f}},
$$

where $T_{s}$ is the structural reverberation time of the $i$ or $j$ element in seconds, $S$ is area of the $i$ or $j$ element in $\mathrm{m}^{2}, f$ is the band central frequency in $\mathrm{Hz}, f_{\text {ref }}$ is the reference frequency of the $1000 \mathrm{~Hz}$ value and $c_{o}$ is the air sound velocity in $\mathrm{m} / \mathrm{s}$.

As specified in the standard, using Eqs. (1) and (2), it is possible to obtain the $K_{i j}$ value from the measuring of the difference of the velocity levels in both directions and the structural reverberation time of both the elements. To obtain the difference of the velocity levels, the standard establishes a set of geometric constraints. Some of the conditions specified are:

- 3 excitation points and 9 transducer positions (3 per excitation point) must be chosen on each element.

- The positions should be distributed randomly but not symmetrically.

- The positions of the transducers and excitation points must meet the following minimum distances:

$-0.5 \mathrm{~m}$ between the excitation points and the limits of the element to be tested,

$-1 \mathrm{~m}$ between the excitation points and the associated transducer positions,

- $0.5 \mathrm{~m}$ between each transducer position.

In addition, the determination of the difference of the velocity levels requires accomplishing the coupling conditions between the elements forming the union; if these conditions are not met, the data obtained from the in situ measurements are not representative of the energy distribution between these elements a are not valid for obtaining the vibrational reduction index. This coupling condition is assessed in the following inequality:

$$
D_{v, i j} \geq 3-\log \left(\frac{m_{i} f_{c j}}{m_{j} f_{c i}}\right)[\mathrm{dB}],
$$

where $m_{i}, m_{j}$ are surface densities of the $i$ and $j$ elements in $\mathrm{kg} / \mathrm{m}^{2}$ and $f_{c i}, f_{c j}$ are their respective critical frequencies in Hertz.

As an alternative, the empirical equations proposed in the EN 12354 standard allow an easier calculation of the $K_{i j}$ factors for the analysis of common types of joints and makes the $K_{i j}$ factors depend on the surface densities of the elements connected to the union.
These expressions can be used only for the case of unions in which elements on both sides of the junction are in the same plane and have the same mass. Thus only two mass ratios can be defined. Figures 1a and $1 \mathrm{~b}$ show rigid cross-joints and T-joints, respectively.

a)

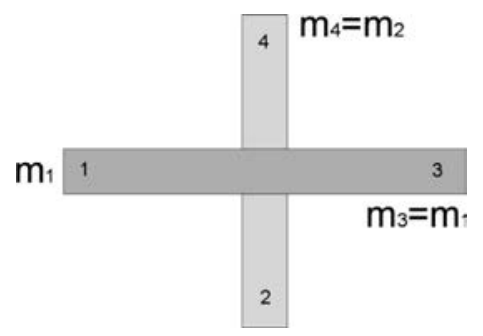

b)

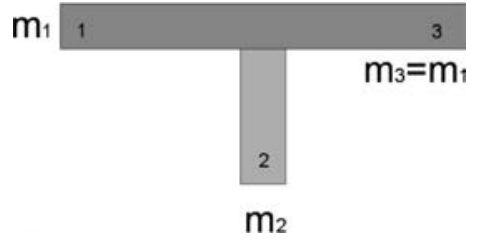

Fig. 1. a) Rigid cross-joint, b) rigid T-joint.

In the case of a cross rigid connection, the following relations are defined for the vibrational reduction index:

$$
\begin{aligned}
& K_{13}=8.7+17.1 M+5.7 M^{2}[\mathrm{~dB}] ; 0 \mathrm{~dB} / \text { octave } \\
& K_{12}=8.7+5.7 M^{2}\left(=K_{23}\right)[\mathrm{dB}] ; 0 \mathrm{~dB} / \text { octave. }
\end{aligned}
$$

These expressions are given in terms of the magnitude $M$ defined as:

$$
M=\log \left(m_{\perp i}^{\prime} / m_{i}^{\prime}\right),
$$

where $m_{i}^{\prime}$ is the superficial density of the $i$ element in the $i j$ transmission path, and $m_{\perp i}^{\prime}$ is the superficial density of the other element, perpendicular to $i$, that forms the union. The calculation of these masses takes into account only the base material connected to the adjacent construction elements, so the masses of the coatings, such as the floating floors or suspended ceilings, must be excluded. The magnitudes given in (4) and (5) are not frequency dependent. In general, the standard states that the energy transfer is less frequency dependent in the range from $125 \mathrm{~Hz}$ to $2 \mathrm{kHz}$, so it is considered to be $0 \mathrm{~dB} /$ octave.

The vibrational reduction in the joints can be significantly improved with flexible intermediate layers, as depicted in Fig. 2. For that case, the improvement referred to a rigid joint, $\Delta_{1}$, occurs beyond a frequency $f_{1}$ that depends on the flexible element thickness $\left(t_{1}\right)$ and the elastic modulus $\left(E_{1}\right)$. 
a)

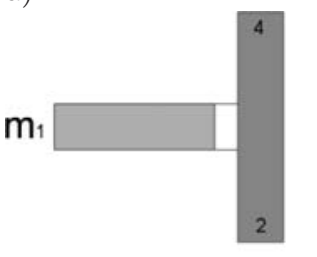

$\mathrm{m}_{2}=\mathrm{m}_{4}$

c)

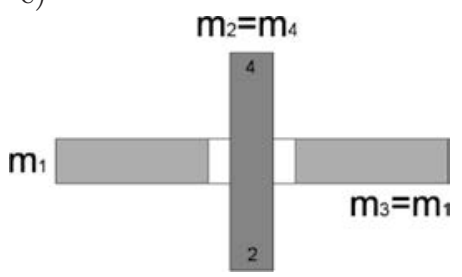

Fig. 2. T and cross-joints with flexible interlayers.

This correction factor $\Delta_{1}$ represents a frequency dependence, that exists why the standard proposes a set of equations to determine the $K_{i j}$ factors in the presence of an intermediate flexible layer. These are:

$$
\begin{aligned}
K_{13}= & 5.7+14.1 M+5.7 M^{2}+2 \Delta_{1}[\mathrm{~dB}], \\
K_{24}= & 3.7+14.1 M+5.7 M^{2}[\mathrm{~dB}] ; \\
& -4 \leq K_{24} \leq 0[\mathrm{~dB}] ; \quad 0 \mathrm{~dB} / \text { octave, } \\
K_{12}= & 5.7+5.7 M^{2}+\Delta_{1}\left(=K_{23}\right)[\mathrm{dB}], \\
\Delta_{1}= & 10 \log \left(f / f_{1}\right)[\mathrm{dB}] \quad \text { when } f>f_{1}, \\
f_{1}= & 125[\mathrm{~Hz}] \quad \text { if } \quad E_{1} / t_{1} \approx 100 \mathrm{MN} / \mathrm{m}^{3} .
\end{aligned}
$$

The estimation given in (10) is a global value for some typical joints, where $E_{1}$ is the interlayer Young elastic modulus and $t_{1}$ its thickness. The parameters determining the dynamic stiffness of an elastic band are the nature of it (in terms of elastic properties) and its thicknes. Thus, for the same material, the Young's modulus remains constant and therefore, when the thickness increases, the dynamic stiffness decreases, and a more efficient damping effect occurs. However, the EN 12354 standard is rather vague concerning the calculation of $f_{1}$, which means that it can be conceived as a function of the shear modulus, the flexible element thickness and the connected element densities $\rho_{1}$ and $\rho_{2}$. The $f_{1}$ dependence with respect to those parameters can be written as:

$$
f_{1} \propto\left(\frac{G}{t_{1} \sqrt{\rho_{1} \rho_{2}}}\right)^{1.5} .
$$

Based on theoretical and experimental data, PEDERSEN (1995) proposed that this crossover frequency $f_{1}$ should be written as:

$$
f_{1}=2.510^{-6}\left(\frac{\sqrt{\rho_{1} \rho_{2}}}{G} t_{1} \frac{l}{w}\right)^{-3 / 2},
$$

where $\rho_{1}$ and $\rho_{2}$ are the volumetric densities of the elements that compose the union, $G$ is the shear modulus, $t_{1}$ the thickness of the elastic layer, $l$ is the common length of the elements forming the union and $w$ the common length of the elastic element in the union. It is also assumed that for frequencies above $f_{1}$ the improvement of the elastic layer increases with frequency, while at lower frequencies no improvement can be appreciated.

For the frequencies $f<f_{1}$, vibrational reduction can be calculated as if there were no intermediate layer. Above $f_{1}$ the vibrational reduction increases by $10 \log \left(f / f_{1}\right)$ with an interlayer in the union, and by $20 \log \left(f / f_{1}\right)$ when two interlayers are interposed in the transmission path (PEDERSEN, 1995; SCHNEIDER, Fischer, 2005; CRISPIN et al. 2004).

\section{Numerical analysis}

\subsection{Methodology}

As a starting point, consider the vibration reduction index of a structure given in Eq. (1). Considering $S_{i}$ and $S_{j}$ as the areas of the $i$ and $j$ elements, $c_{o}$ as the air sound velocity and $T_{s i}, T_{s j}$ the structural reverberation times of each element, this equation can be rewritten as:

$$
\begin{aligned}
& K_{i j}=\overline{D_{v, i j}} \\
& +10 \log \left(\frac{l_{i j}}{\sqrt{\left[\frac { 2 . 2 \pi ^ { 2 } S _ { i } } { c _ { o } T _ { s i } } \sqrt { \frac { f _ { \mathrm { ref } } } { f } } \left[\frac{2.2 \pi^{2} S_{j}}{c_{o} T_{s j}} \sqrt{\frac{f_{\mathrm{ref}}}{f}}\right.\right.}}\right) .
\end{aligned}
$$

To reduce the number of analyzed variables in our analysis, a constant area $S$ for all surfaces $\left(S=S_{i}=\right.$ $\left.S_{j}\right)$ is considered; additionally, the structural reverberation time $T_{s}$ will be defined as a function of frequency and loss factor $\eta_{\text {tot }}$ :

$$
T_{s}=\frac{2.2}{f \eta_{\mathrm{tot}}}
$$

where $\eta_{\text {tot }}$ can be obtained using the relationship:

$$
\eta_{\mathrm{tot}}=\eta_{\mathrm{int}}+\frac{m^{\prime}}{485 \sqrt{f}}
$$

with $m^{\prime}$ the superficial density, in $\mathrm{kg} / \mathrm{m}^{2}$. This formula applies only if $m^{\prime}<800 \mathrm{~kg} / \mathrm{m}^{2}$ according to EN 12354 .

Introducing these relations, Eq. (13) can be simplified and the vibrational reduction index $K_{i j}$ rewritten as:

$$
K_{i j}=\overline{D_{v, i j}}+10 \log \left(\frac{l_{i j} c_{o}}{\pi^{2} S \sqrt{\eta_{i} \eta_{j} f f_{\mathrm{ref}}}}\right) .
$$


Defining a relational term between loss factors of the analyzed surfaces, $\tau=\eta_{i} / \eta_{j}$, Eq. (16) becomes:

$$
K_{i j}=\overline{D_{v, i j}}+10 \log \left(\frac{l_{i j} c_{o}}{\pi^{2} S \sqrt{f_{\mathrm{ref}}}}\right)-10 \log \left(\eta_{j} \sqrt{\tau f}\right) .
$$

Later in this paper, the third term of Eq. (17) will be the main focus of our analysis.

\subsection{Numerical model description}

The numerical experiment carried out follows the testing methodology specified in the ISO 10848 standard regarding the placement of excitation sources and measurement transducers, as well as the models size. The materials simulated have a density of $2400 \mathrm{~kg} / \mathrm{m}^{3}$, a Young's modulus of $32 \mathrm{GPa}$ and a Poisson coefficient of 0.22 . The simulated models have thicknesses of 10 , 20 and $30 \mathrm{~cm}$ and an internal loss factor of 0.01 . The elastic layer has been chosen meeting the condition imposed by (10) with a loss factor of 0.2 .

A constant force of $1 \mathrm{~N}$ is applied in the considered frequency range. Trying to emulate the standard conditions, a distance of $1 \mathrm{~m}$ has been left between the applied force points and of $0.5 \mathrm{~m}$ between the measurement positions.

From simulations, the velocities at different $i$ and $j$ positions are obtained. From this data and according to the standard, the averaged velocity level difference is calculated.

a)

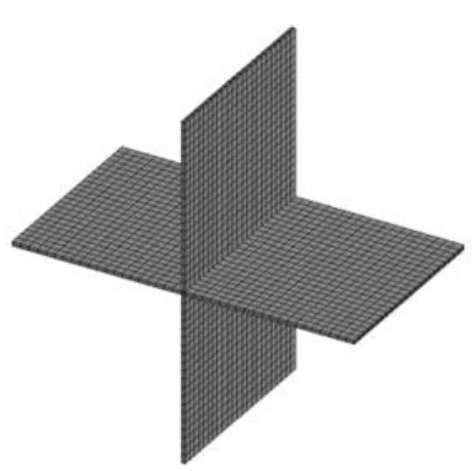

b)

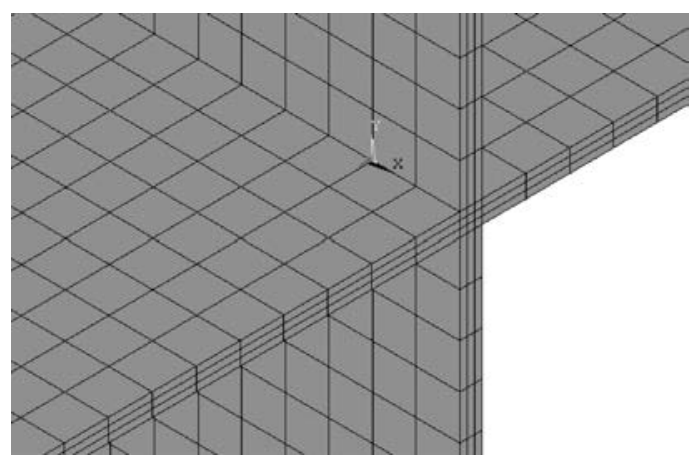

Fig. 3. Schematic of the finite element model: a) global view of the cross-joint, b) model details.
The numerical finite element model is built with the ANSYS ${ }^{\circledR}$ software using about 6000 elements (using high order 3-D 20-node solid elements that exhibit a quadratic displacement behavior; there are three degrees of freedom per node: translations in the nodal $\mathrm{x}$, $\mathrm{y}$ and $\mathrm{z}$ directions). The materials with linear behavior are modeled and with a constant loss factor. The element size is defined so that it is less than a tenth of the wavelength. Regarding the boundary conditions, the three translational movements (three degrees of freedom) of all nodes located at both ends of the model are prescribed as null. To account for the complexity of the model, it suffices to say that 29808 nodes have been used for the simplest cross-joint model corresponding to $3 \times 29808=89424$ degrees of freedom.

In addition to those models, and in order to compare cross-joint models with the case where a flexible interlayer is assembled, additional models were implemented for each of the joints under study. Some details of the mesh employed for these models are shown in Fig. 4.

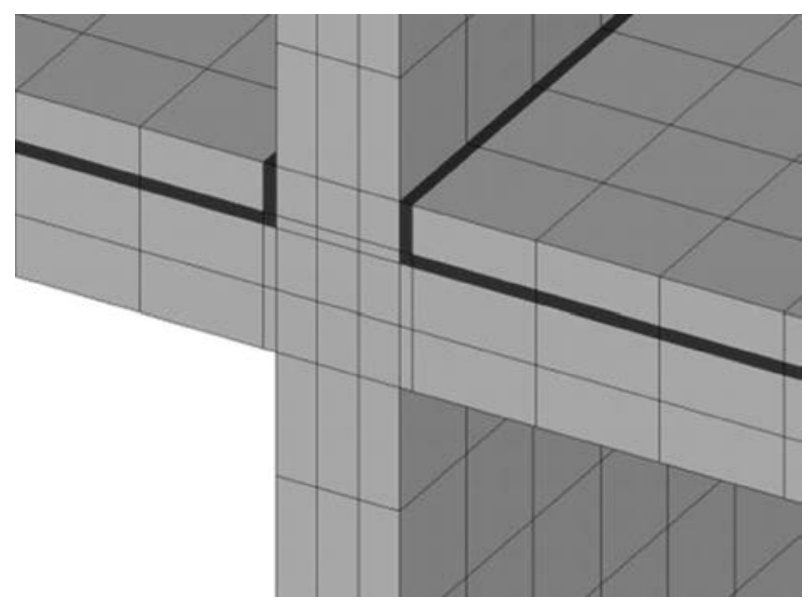

Fig. 4. Model of a cross-joint with flexible interlayer.

As shown in the figure above, the flexible interlayer is not located as specified in EN 12354; although this is a more realistic configuration, there are no estimated equations for these kinds of joints when the interlayer does not cover the entire joint and extends to the whole floor surface (so the pressure reduction index equations in the Annex C of EN 123542 are obviously not directly applicable when evaluating $\left.K_{i j}\right)$.

Since the simulations were performed for several combinations of thicknesses, the mass and geometry effect on structural vibration was also compared. Additionally, as part of our interest was to contrast the numerical results with those of the EN 12354 estimations and accomplish the measurement conditions specified in ISO 10848, the simulations were carried out considering several excitation points. 


\subsection{The numerical $K_{i j}$ for rigid cross-joints}

A first set of results is shown in Fig. 5 that corresponds to those obtained for rigid cross-joints. Having calculated velocity level differences as an average of several source and sensor points and meeting the relevant restrictions, we have obtained the vibration reduction index for the flanking transmission and compare them with those obtained following the EN 12354 standards.

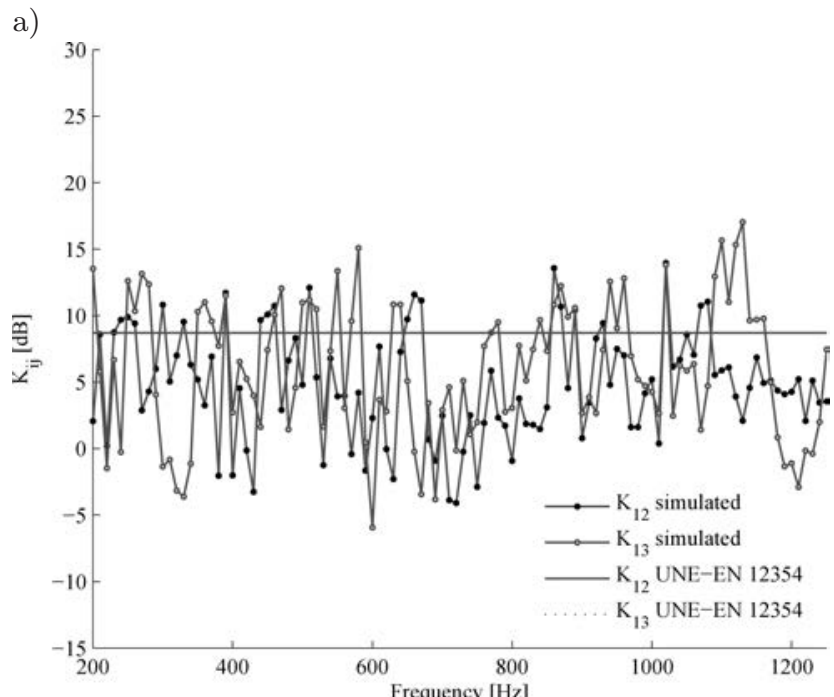

b)

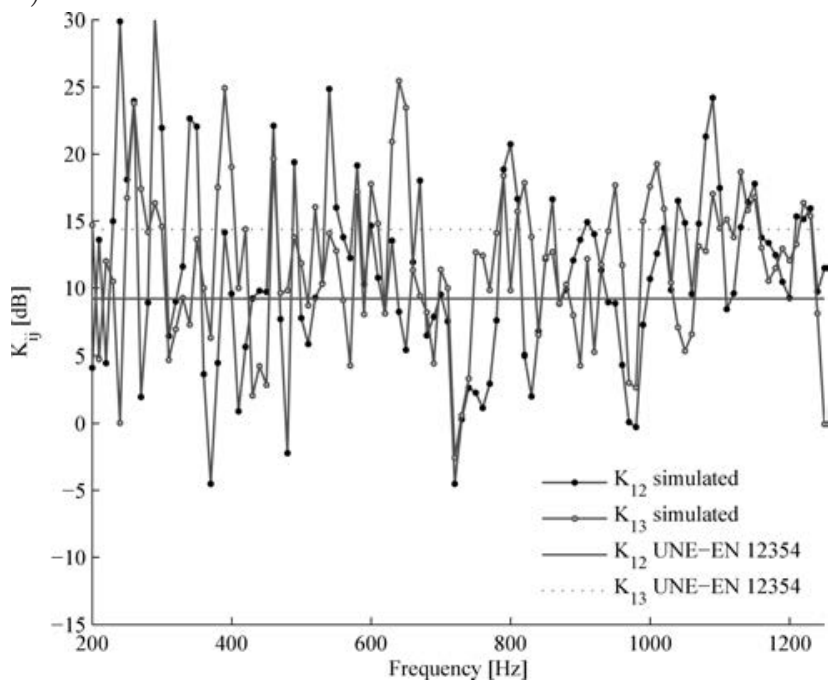

Fig. 5. Vibration reduction index $K_{i j}$ for rigid cross-joints: a) same thickness $(M=0)$, b) different thickness $(M \neq 0)$.

In Fig. 6, results obtained when evaluating the same model but considering a floating slab with a flexible interlayer are displayed.

There should be highlighted the fact that, like in the case of rigid junctions, some of the vibration reduction index values obtained are lower than the $K_{i j \text {,min }}$ specified in EN 12354 (Eq. (23)) and should be therefore neglected.
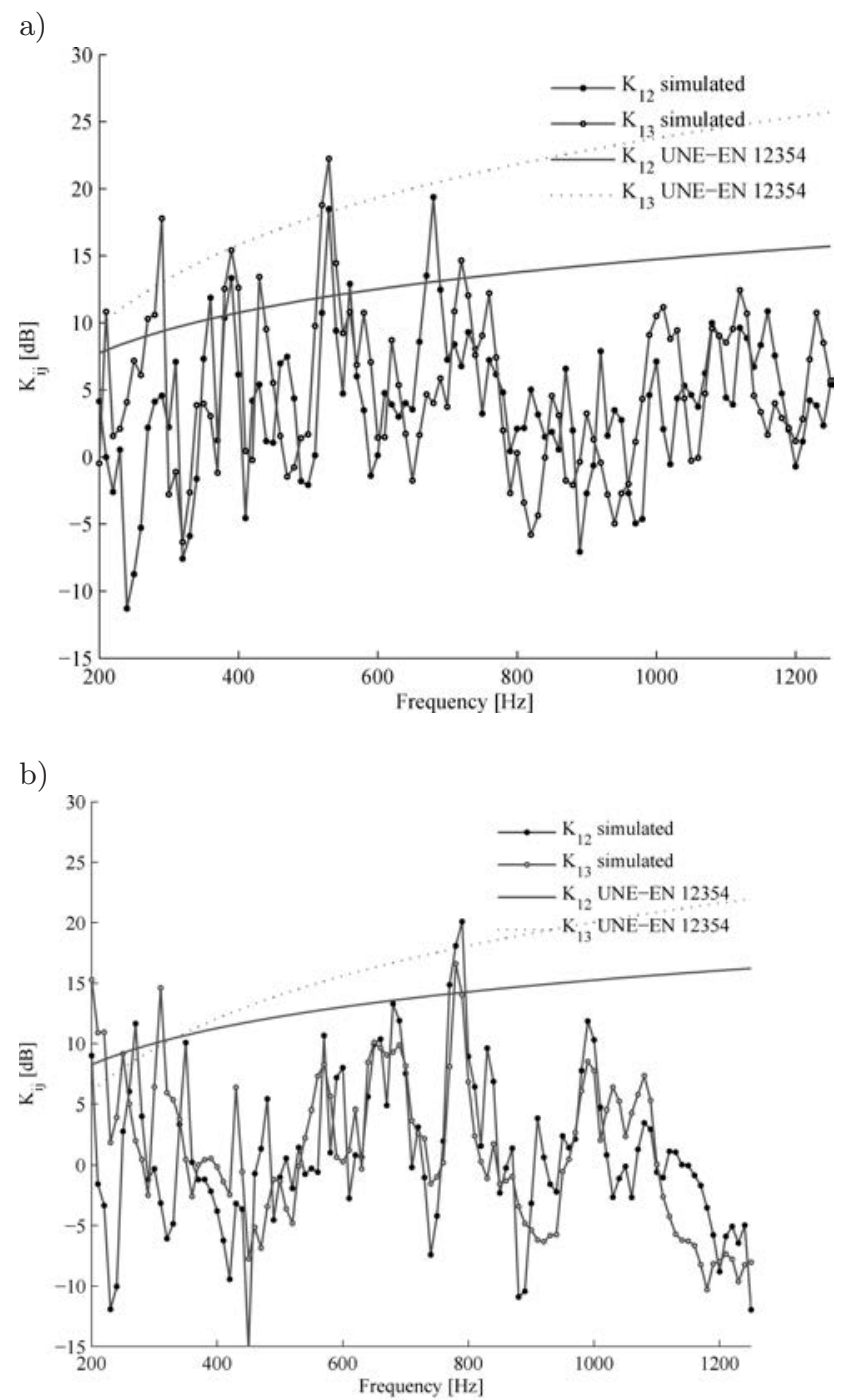

Fig. 6. Vibration reduction index $\mathrm{K}_{i j}$ for rigid cross-joints with flexible interlayer: a) same thickness $(M=0)$, b) different thickness $(M \neq 0)$.

\section{Discussion}

To analyze the differences between the numerical and simplified results presented before and allow for a clearer understanding of the involved methodologies, let us focus firstly on the behavior of rigid unions. For that case, the results in Fig. 5 have clearly shown that the simulated behavior, making use of a FEM model, reveal a very complex behavior even for the simplest model with strong variations throughout the frequency domain.

In order to make the simulation results and the empirical equations of EN 12354 converge, an adjustment of the first of them is proposed. Assuming that the $i$ and $j$ elements that take part in the junctions evaluated have an equivalent absorption length $l_{i j}=4.5 \mathrm{~m}$ and the same surface $S=18 \mathrm{~m}^{2}$, we define a "coupling factor" $\tau$ that relates the total loss factors of the 
a)

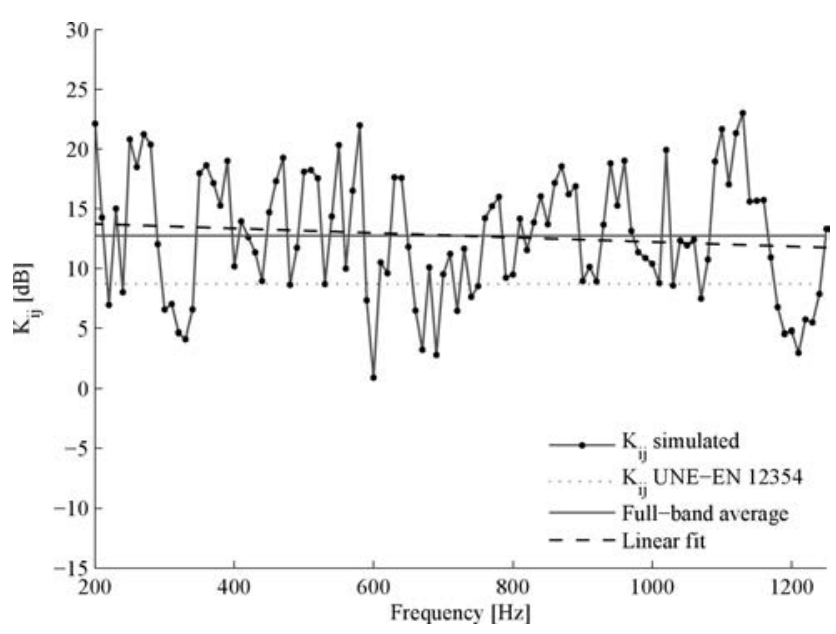

c)

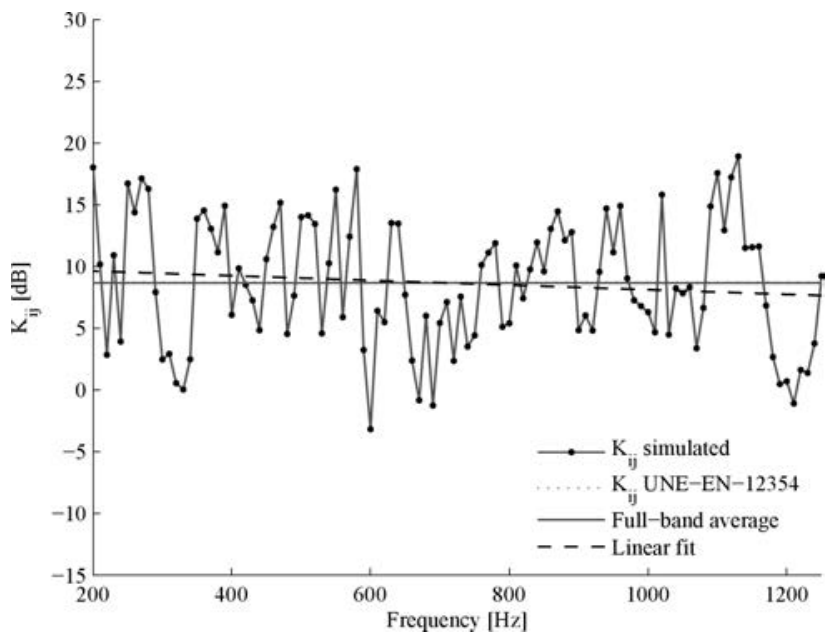

b)

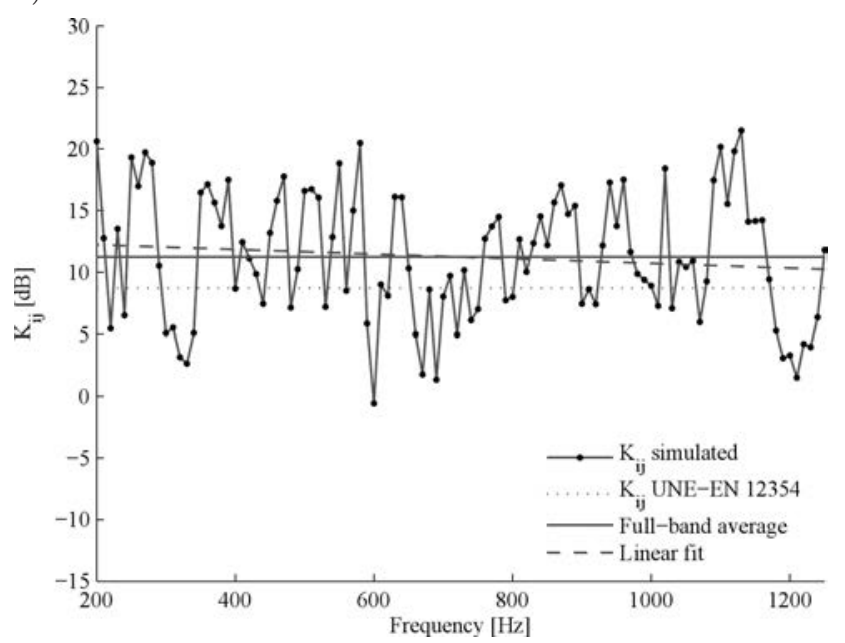

d)

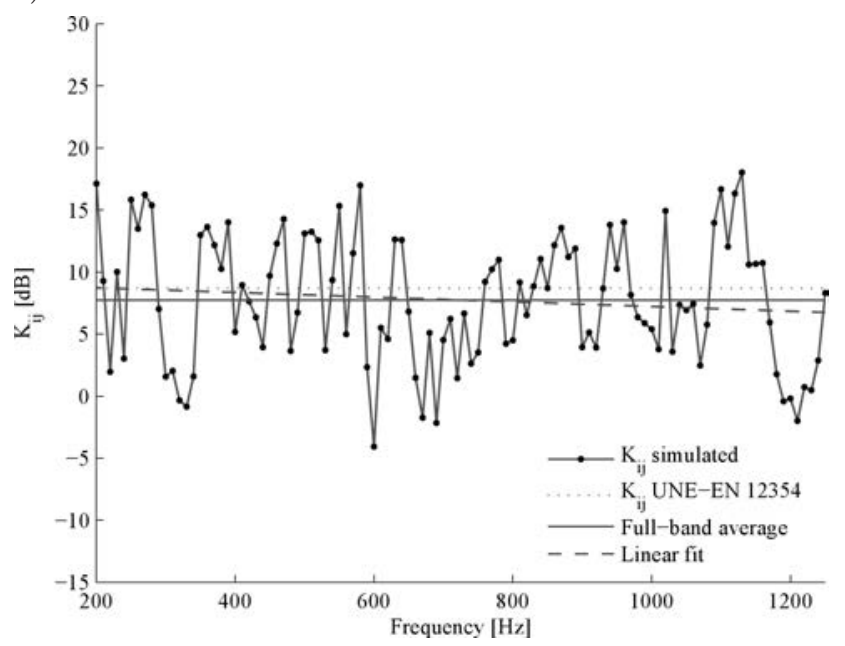

Fig. 7. Vibration reduction index $K_{i j}$ for rigid cross-joints with different $\tau$ values: a) $\tau=0.4$, b) $\tau=0.8$, c) $\tau=2.6$ (optimum value), d) $\tau=4.0$.

$i$ and $j$ elements $\tau=\eta_{i} / \eta_{j}$. For this case, considering $c_{o}=343 \mathrm{~m} / \mathrm{s}$ and $f_{\text {ref }}=1000 \mathrm{~Hz}$, Eq. (17) becomes:

$$
K_{i j}=\overline{D_{v, i j}}-5 \log \left(13.25 \tau \eta_{j}^{2} f\right)
$$

Evaluating this equation for different values of $\tau$ in a rigid cross-joint transmission flank, for which we assume a value of $\eta_{j}=0.01$, an inverse relation between the parameter $\tau$ and the resulting $K_{i j}$ can be observed in Fig. 7.

For this test case, the ascribed $\tau=2.6$ produces the best approximation between the numerical and the simplified approaches. Unlike the case of flexible interlayer, the standard EN 12354-2 does not consider any frequency dependence of the rigid junctions. To allow a more detailed analysis, an additional frequency dependent term $T$ is added to Eq. (18):

$$
K_{i j}=\overline{D_{v, i j}}-5 \log \left(13.25 \tau \eta_{j}^{2} f\right)-T \text {. }
$$

This logarithmic term $T$ can be expressed as a frequency dependent variable $\gamma$ that multiplies the frequency:

$$
T=5 \log (\gamma f)
$$

By introducing this term in the previous equation, the final expression is obtained as follows:

$$
K_{i j}=\overline{D_{v, i j}}-5 \log \left(13.25 \gamma \tau \eta_{j}^{2} f^{2}\right) .
$$

Therefore, replacing the previous $\tau$ and $\eta_{j}$ values that allow for the best fit between both the models, and determining the frequency-dependent $\gamma$ values, the convergence of the simplified formulas of the EN 12354 standard and the results computed using the FEM model can be achieved making use of the curve given in Fig. 8.

Let us now apply the same methodology to analyze the case of a cross-joint including a flexible interlayer. Once again, we make use of the average re- 


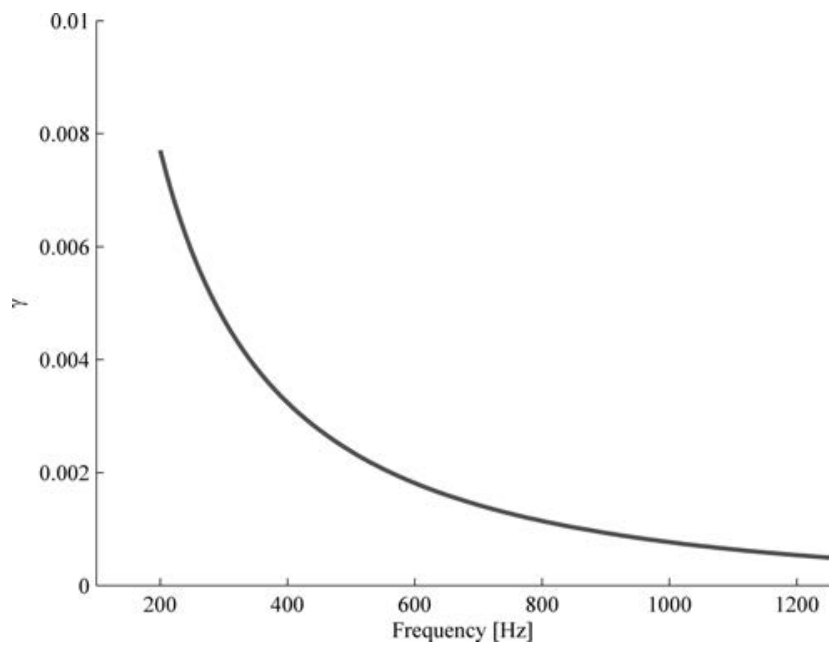

Fig. 8. Values of the $\gamma$ parameter that better fits for an optimal convergence with the $K_{i j}$ given by EN 12354 for the case of a rigid cross-joint.

sults computed from the FEM model considering several impact and measurement points throughout the frequency spectrum. For this case, the best fit between the full-band averages applied to the FEM results and to the curve predicted using EN 12354-2 would occur for $\tau=0.008$ and $\eta=0.01$ as shown in Fig. 9 .

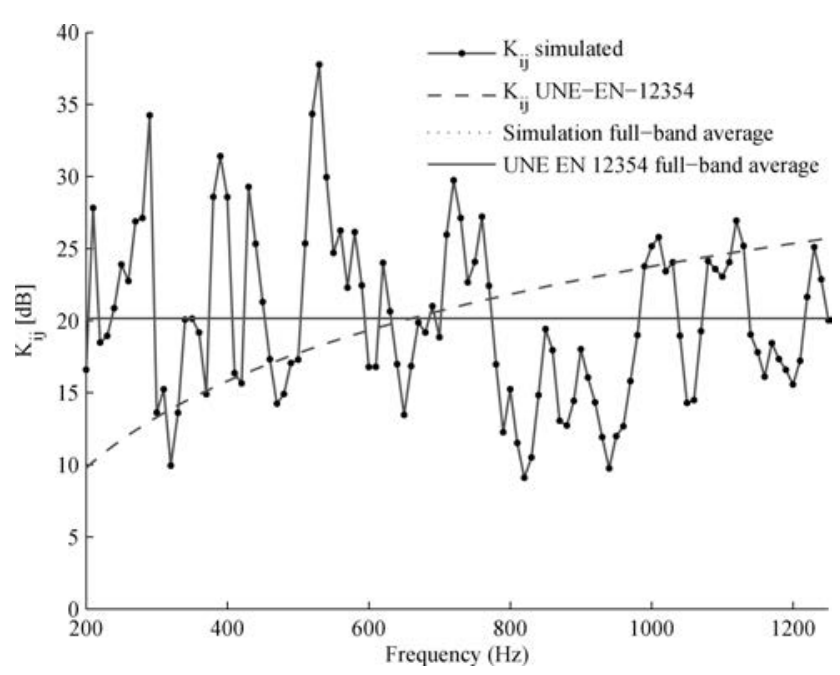

Fig. 9. Results for a cross-joint with a floating slab and elastic interlayer. "Best-fit" results are obtained considering $\tau=0.008$.

As in the previous scenario, it becomes necessary to compute the difference between the linear fit of the FEM results and the normative curve and to find the corresponding values of $\gamma$ that allow making the numerical results converging with the simplified curve. For this case, the computed values of $\gamma$ that allow for this optimal fit are given in Fig. 10 as a function of frequency.

The two examples presented allow to conclude that the behavior of structural joint is indeed complex and

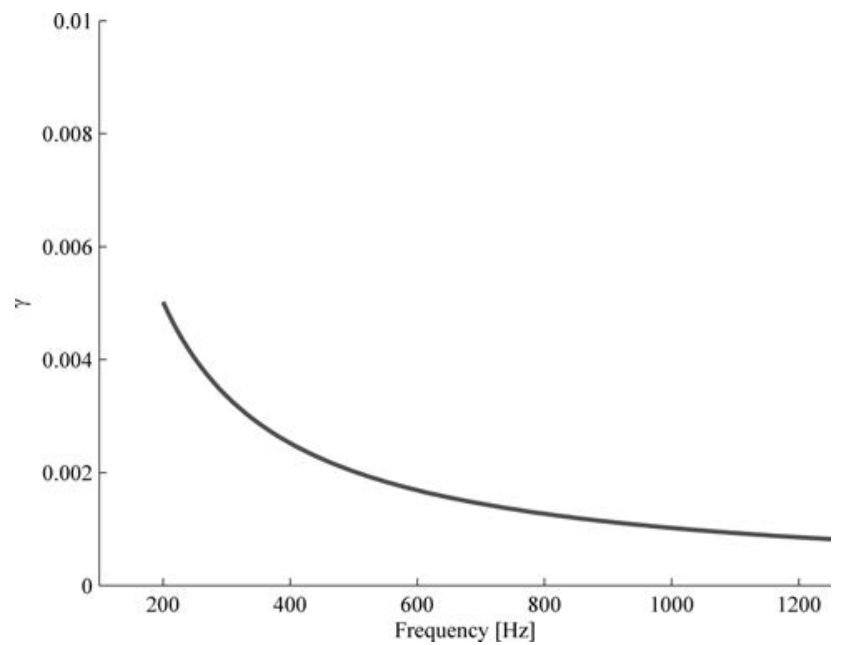

Fig. 10. Values of the $\gamma$ parameter that better fits for an optimal convergence with the $K_{i j}$ values given by EN 12354 for the case of a cross-joint with flexible interlayer.

strongly frequency-dependent. This complex behavior can hardly be represented just by the simple equations proposed in EN 12354-2, and thus special care must be taken in its analysis. However, those equations may be a starting point to predict this behavior but they must be adapted to incorporate the specificity of each individual structural configuration. The curves presented above, revealing a frequency dependent $\gamma$ parameter, may indicate a possible strategy to perform this correction although they should be interpreted as representative only for the cases tested.

\section{Final remarks}

The present work addressed the issue of structural vibration transmission in the presence of structural joints. A numerical study was performed for a number of typical constructive scenarios, for which FEM results were compared with those provided by the standard EN 12354-2. The presented examples allowed to conclude that the behavior of a structural joint in terms of vibration transmission is complex and depends strongly on the frequency, and that this is hardly well represented by the simple expressions proposed in the standard. A simple methodology to estimate the difference between numerical and normative predictions was presented allowing the calculation of an adaptation term to make the two approaches converge. This term was found to be solution-dependent and thus should be evaluated for each structural reality.

\section{Acknowledgment}

This work was supported by the Ministry of Science and Innovation (Spain) under the Project BIA201017723 and by the University of Alicante under the Project GRE10-29. 
This paper represents an improvement and expansion of a paper presented at the 42th CONGRESS OF ACOUSTIC-TECNIACÚSTICA ${ }^{\circledR}$ 2011, Iberian Meeting on Acoustics and European Symposium on Environmental Acoustics and Sustainable Building Acoustics, organized by the Sociedad Española de Acústica (SEA), the Acoustics Laboratory of the University of Extremadura, and the Sociedade Portuguesa de Acústica (SPA), and with the support of the European Acoustics Association (EAA) and the International Commission for Acoustics (ICA).

\section{References}

1. Brunskog J., Lhomond A., Ohlrich M. (2007), Attenuation and flanking transmission in lightweight structures, Proceedings of the 19th International Congress on Acoustics, Madrid, Spain.

2. Brutel-Vuilmet C., Villot M., Guigou-CarTER C., JEAN P. (2006), Measurement of the sound reduction index as a function of the incidence angle by two different methods, Building Acoustics, 13, 1, 3348.

3. Brutel-Vuilmet C., Villot M., Guigou-Carter C. (2007), Use of the NAHtechnique to assess the effects of the incidence angle on the reduction index, Acta Acustica, 93, 3, 364-374.

4. Clasen D., Langer S. (2007), Finite Element Approach for Flanking Transmission in Building Acoustics, Building Acoustics, 14, 1, 1-14.

5. Craik R. (2001), The contribution of long flanking paths to sound transmission in buildings, Applied Acoustics, 62, 1, 2946.

6. Crispin C., Mertens C., Blasco M., Ingelaere B., VAn Damme M., Wuyts D. (2004), The vibration reduction index Kij: laboratory measurements versus predictions EN 12354-1 (2000), Internoise 2004, The 33rd International Congress and Exposition on Noise Control Engineering, Prague, Czech Republic.

7. EN ISO (2000), 12354-1:2000. Building acoustics - Estimation of acoustic performance of buildings from the performance of elements - Part 1: Airborne sound insulation between rooms, International Organization for Standardization, Geneva.

8. EN ISO (2000), 12354-2:2000. Building acoustics - Estimation of acoustic performance of buildings from the performance of elements - Part 2: Impact sound insulation between rooms, International Organization for Standardization, Geneva.
9. Galbrun L. (2008), The prediction of airborne sound transmission between two rooms using first-order flanking paths, Applied Acoustics, 69, 12, 1332-1342.

10. Gerretsen E. (1979), Calculation of the sound transmission between dwellings by partitions and flanking structures, Applied Acoustics, 12, 6, 413-433.

11. Gerretsen E. (1986), Calculation of airborne and impact sound insulation between dwellings, Applied Acoustics, 19, 4, 245-264.

12. Gerretsen E. (2008), Prediction models for building performance - European need and world wide use, Journal of the Acoustical Society of America, 123, 5, 3189 (one page).

13. ISO (2006), 10848-parts 1, 2 and 3. Laboratory measurement of the flanking transmission of airborne and impact sound, between adjoining rooms.

14. Kling C. (2008), Investigations into Damping in Building Acoustics by Use of Downscaled Models, Ph.D. Thesis, Aachen, Aachener Beiträge zur Technischen Akustik.

15. Mahn J. (2009), Prediction of Flanking Noise Transmission in Lightweight Building Constructions: A Theoretical and Experimental Evaluation of the Application of EN12354-1, Ph.D. Thesis, University of Canterbury, Department of Mechanical Engineering.

16. Maluski S., GiBbs B. (2000), Application of a finiteelement model to low-frequency sound insulation in dwellings, Journal of the Acoustical Society of America, 108, 4, 1741-1751.

17. Maynard J., Williams E., Lee Y. (1985), Nearfield acoustic holography: I. Theory of generalized holography and the development of $N A H$, Journal of the Acoustical Society of America, 78, 4, 1395-1413.

18. Metzen H.A. (1999), Accuracy of CEN-prediction models applied to German building situations, Building Acoustics, 6, 3-4, 325-340.

19. Pedersen D.B. (1995), Estimation of Vibration Attenuation through Junctions of Building Structures, Applied Acoustics, 46, 3, 285-305.

20. Santos P., Tadeu A. (2002), Acoustic insulation provided by a single wall separating two contiguous tunnels via BEM, Journal of Sound and Vibration, 257, 5, 945965.

21. Schneider M., Fischer H-M. (2005), Flanking transmission of masonry building elements with flexible interlayer, ForumAcusticum 2005, Budapest, Hungary. 